\title{
Case-Based Reasoning for Flight Conflicts Resolution in En-Route Short Term Conflict Alert Situation
}

\author{
Man Liang ${ }^{1,2, *}$ \\ ${ }^{I}$ College of Civil Aviation, Nanjing University of Aeronautics \& Astronautics, Nanjing, China \\ ${ }^{2}$ College of Air Traffic Management, Civil Aviation University of China, Tianjin, China
}

\begin{abstract}
This paper introduces a knowledge-driven approach for solving short range conflicts in En-route STCA situation in order to assist the air traffic controller to handle emergency and ensure the safety. Instead of applying classic conflict resolution approach, case-based reasoning theory and technology is used to transfer the controllers' knowledge and experience to automate conflict resolution. Firstly on the basis of analysis of the controllers' conflict resolution knowledge with domain experts, a description of case is well established in a structural framework which has two layers, the first layer with 4 Slot features and the second layer with 19 Side features. Secondly, in case retrieval, the source cases are firstly filtered against constraint parameters then a nearest neighbor algorithm and global similarity measure based approach is applied to calculate the degree of similarity between the target case and the source cases. Finally, two scenario tests are designed to verify the performance of CBR-based conflict resolution model. The results indicate that this method is consistent with the solutions recommended by air traffic control experts and has a good effect in application.
\end{abstract}

Keywords: Air traffic control, Case based reasoning, Decision support, Flight conflict resolution.

\section{INTRODUCTION}

Maintaining the safety, efficiency and orderliness of air transport is the overall goal of air traffic control (ATC) operation for civil aviation. Controllers' conflict resolution skills are the key to ensure flight safety. Along with steady growth in traffic flow, there has been a dramatic increase in controller workload, and ATC's unsafe actions caused by human errors are occurring more and more frequently. A reliable and efficient system for resolving conflicts automatically has been proposed as a key component for the next generation air traffic control system.

Given the importance of flight conflict resolution, scholars have applied different kinds of approaches to study it, such as, mathematics, operations research, probability theory, cybernetics and computational theory [1,2]. Analyzed the conflict resolution for aircraft in free flight, under the precondition of not changing the safety level, from the perspective of lowering fuel consumption $[3,4]$. Took into account the speed uncertainties and allowed the aircraft to fly on direct routes, solved every conflict on a busy day, and gave each aircraft its requested flight level and departure time [5]. Studied RR3D algorithms for geometric optimization of flight conflicts [6]. Modeled speed and heading maneuver dynamics in air traffic conflict resolution on flight level, and later [7] introduced an algorithm for computing vertical

\footnotetext{
*Address correspondence to this author at the College of Civil Aviation, Nanjing University of Aeronautics \& Astronautics, 210016, Nanjing, China; E-mail:m-liang@cauc.edu.cn
}

resolution maneuver to resolve imminent air traffic conflicts in which loss of separation could occur within two minutes.

Instead of assuming predefined resolution types, either horizontal or vertical, the controllers, on the other hand, choose horizontal, altitude, or speed maneuvers to resolve conflicts, and adapt their choice based on the characteristics of the conflict encounter and on other factors. Furthermore, classic resolution algorithms have been derived on the assumption that both aircraft in conflict are flying at constant altitude and speed along straight-line paths. However, analysis of actual operations shows that such conflicts constitute less than half of all conflicts encountered in complex en route airspace [8].

The Automated Airspace Concept (AAC) launched in 2001 is with two independent systems for conflict detection and resolution [9]. One element is Autosolver designed to handle strategic conflicts predicted to occur in 2 to 20 minutes. The other element is TSAFE (Tactical Separation Assured Flight Environment) designed to handle tactical separation with times to loss of separation of less than 2 minutes. This system can automatically detect conflict, generate a conflict resolution strategy and transmit the instruction to aircraft via data link [10]. TSAFE is different from TCAS (Traffic Alert and Collision Avoidance System) which is installed in commercial aircrafts. It will act as a powerful decision support tool for controllers in near-loss of separation emergency situation, and at that moment, controllers might lose the situational awareness and perform badly due to heavy stress, even though they have been trained in 


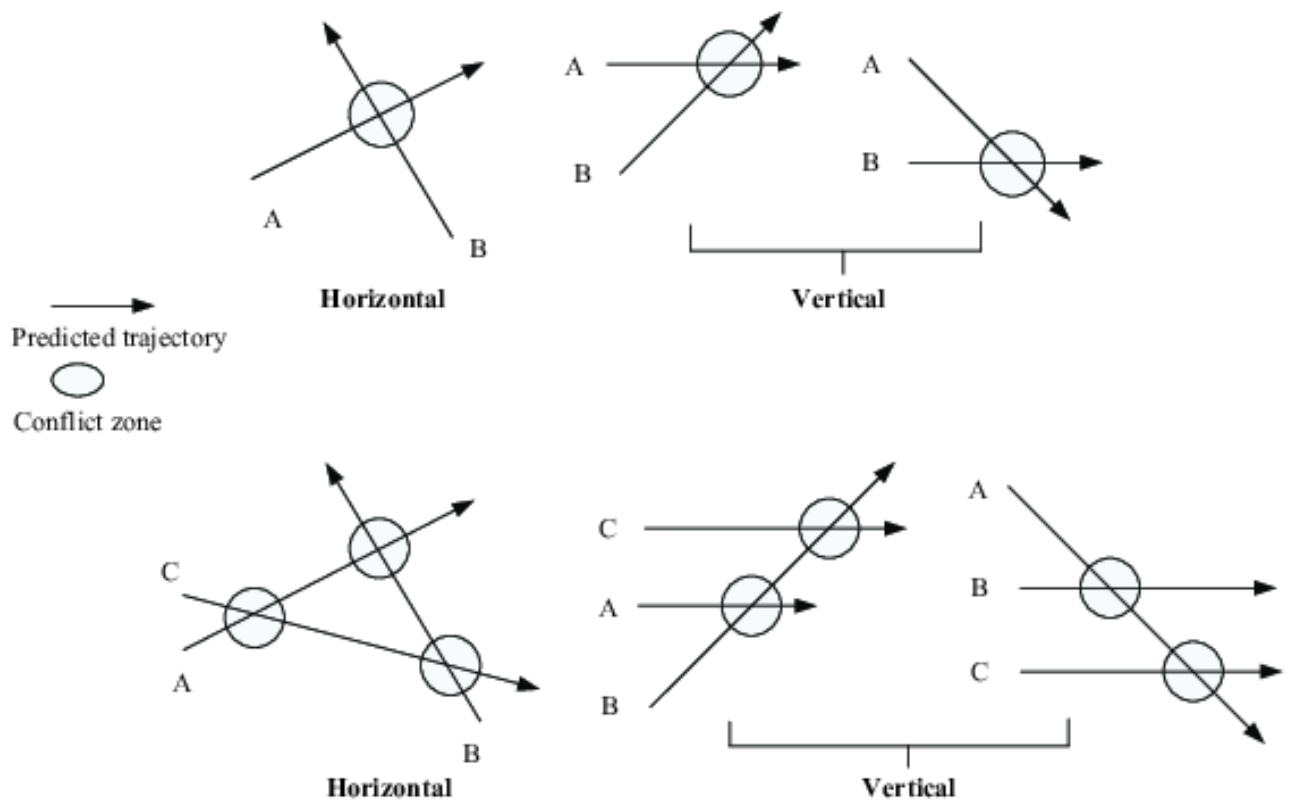

Fig. (1). Conflicts between two aircraft and between multi-aircraft.

the simulator. The algorithm and operational concept in TSAFE for resolving short-range conflicts showed in [11] is by avoiding maneuvers consisting of turns in horizon to a specified heading followed by straight-line flight. On the other hand, there is usually a Short Term Conflict Alert (STCA) for controllers when there is a near loss of separation emergency, and those with experiences and good psychological quality will handle the emergency successfully, but others may not be in that state. Thus, it is necessary to save the valuable experience and successful cases to be used in the future, because these mature methods have played a positive role in current ATC operation.

Application of artificial intelligence theory and technology to transfer the ATC knowledge and experience to automate conflict resolution already has a good application in the ATM [12]. Applies a knowledge-based conflict resolution algorithm for terminal area air traffic control advisory generation, and it is proved that a knowledge based conflict resolution which uses a knowledge base to solve complex control problems would be more suitable to generate a resolution of predicted conflict in a manner consistent with controller practice, and will be much better to transfer the expert knowledge to practical application. Rule-based expert system is one of the knowledge-based systems, but expert system based on rule reasoning is inadequate to accommodate the complex environment for conflict resolution. Case-based Reasoning (CBR) is another important knowledge-based solving and learning method in artificial intelligence (AI). Unlike decision trees and neutral network, it is not only a powerful method for computer reasoning but also a pervasive behavior in everyday human problem solving, and by combining with other intelligent computation algorithms it has been successfully applied to an intelligent fault diagnosis
[13], diseases prognosis and diagnosis [14], and the health sciences [15]. Therefore, attempts can be made to apply CBR technology to automated short range conflict resolution in order to reduce controller faults in a STCA situation and improve the level of safety.

This paper presents a case-based reasoning system for automated en-route short range conflict resolution in a STCA situation. The study mainly consists of two aspects: case representation and case retrieval. Firstly, controller's experiences in resolving conflicts are represented as specific knowledge organized with case framework and case features which are carefully analyzed. Secondly, case retrieval is carried out to obtain the best historical case matching the new case through similarity calculation based on nearest neighbor algorithm. Finally, a technical test is conducted through simulation.

\section{CONCEPTS OF EN-ROUTE FLIGHT CONFLICT RESOLUTION}

Flight conflict refers to the situation in which the distance between two or more aircrafts are smaller than the required separation minimum. The value of the applicable separation minimum is dependent on multiple factors, including category of controlled airspace, mode of surveillance and the relative position between aircrafts. Under radar control situation, the minimum horizontal separation for area (en-route) control is normally $10 \mathrm{~km}$ in China and the minimum vertical separation is $300 \mathrm{~m}$ or $1000 \mathrm{~m}$ (depending on flight level) in China. En-route flight conflicts are generally divided into conflict between a pair aircraft and conflict between more than two aircrafts, as shown in Fig. (1) respectively. 


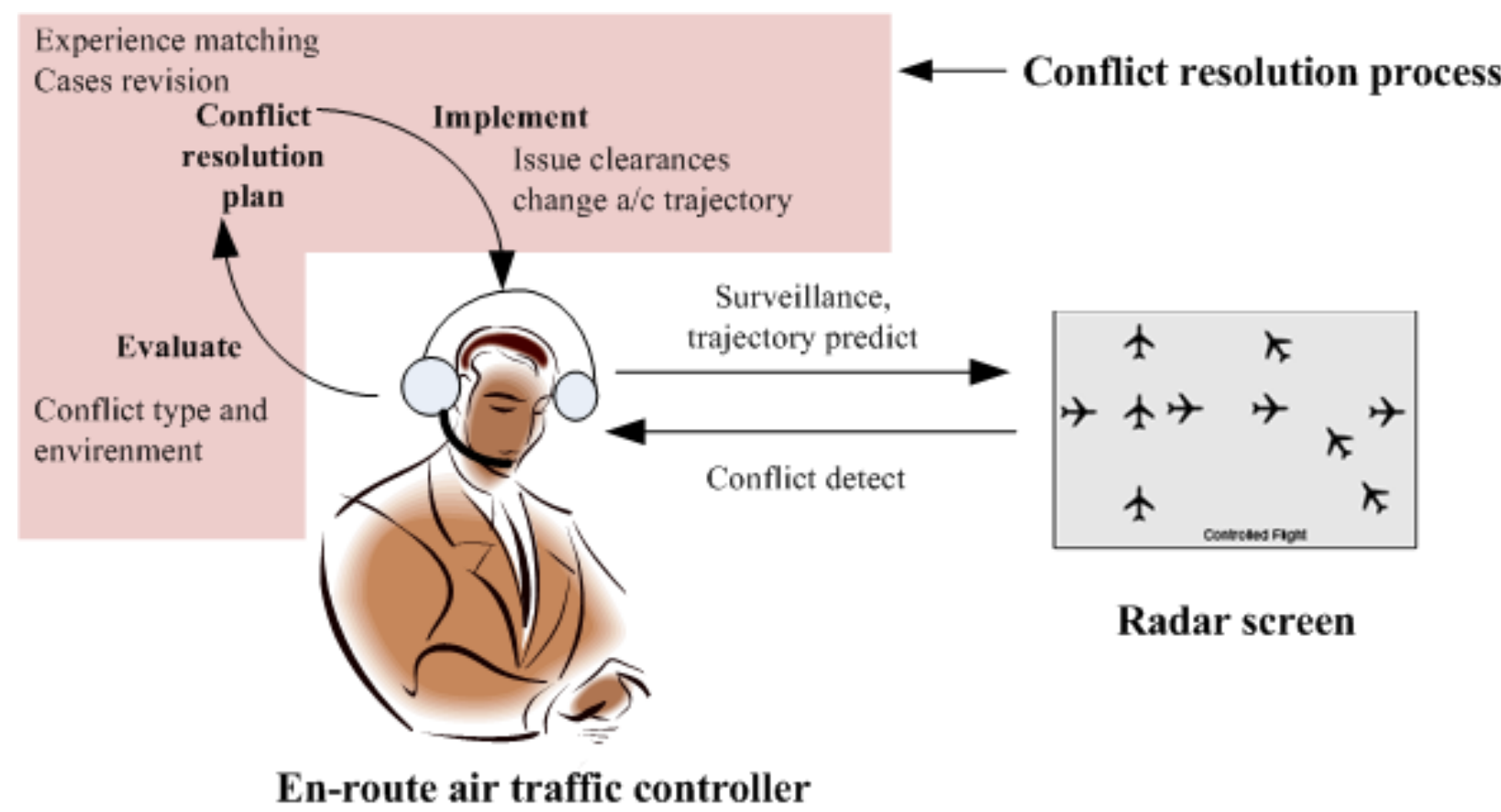

Fig. (2). Traditional En-route ATCO flight conflicts resolution model.

Controller workload is likely to remain the main functional limitation on the capacity of the ATM system [16]. Conflict detection and resolution is the most important task of the controller for ensuring safety of aircraft operation, thus it is the major component of controller workload. As illustrated in Fig. (2), the task of conflict detection and resolution starts with identification of potential conflicts during radar monitoring. Firstly, controllers compare the new case of potential conflict with memory cases relevant to solving it, and then map the solution from the most relevant previous case to the target problem, adapt its solution to fit the new situation. The typical conflict detection and resolution process is shown in Fig. (3). General resolution experience for avoiding aircraft collision is using level change, speed modification and heading adjustment, sometimes even holding to adjust the aircraft's flight trajectory and assuring aircraft safety by providing sufficient lateral or longitudinal separation. In reality, the controller usually uses separation slightly longer than the separation standard due to the lack of precision in predicting the future aircraft trajectory and estimating conflict situation. As the detection of flight conflict and the choice of avoiding maneuver is closely related to many factors, such as the surveillance technology, airspace environment, solving time and human factors, thus in this paper we presuppose the following assumptions: (1) the study environment is radar control; (2) aircraft are in en-route flight period; (3) applied separation minima is: horizontal separation of $20 \mathrm{~km}$ and a vertical separation of $300 \mathrm{~m}$; (4) the conflict resolution time zone refers to about \pm 2 minutes around STCA warning triggering in EUROPECAT system; (5) variations between displayed altitude and position and actual altitude and position, due to periodicity of radar scanning, are not taken into account.

According to the analysis mentioned above, the study approach adopted in this paper is organized as follows. Suppose the conflict between aircraft $i$ and aircraft $j$ is a conflict pair expressed by $C_{i j}$. The time of $C_{i j}$ detected by the controller is $t_{i j}^{d}$, and the time of loss of the separation is $t_{i j}^{c}$. Then, the time available for the controller to solve the conflict $C_{i j}$ is $T_{i j}^{a}=t_{i j}^{c}-t_{i j}^{d}, T_{i j}^{a}$ reflects the degree of urgency for conflict resolution. With respect to multiple flight conflicts involving $n$ aircraft, this global problem may be divided into a set of pairwise problems $\left\{C_{i j}^{1}, C_{i j}^{2}, C_{i j}^{3}, \ldots, C_{i j}^{n}\right\}$ which are prioritized according to the value of $T_{i j}^{a}$. Following this approach, the problem of multiple flight conflicts in en-route flights under a complex environment is simplified by a rolling strategy into the study of cyclic resolution of individual conflict pair.

When the first conflict is identified, we begin to search the most relevant case in the case library by a specific case retrieval algorithm, and then the best candidate case is found through assessment. After that, we adapt or adjust its solution to the new conflict situation. In this process, one aircraft trajectory is modified and then a new trajectory is generated by adopting conflict resolution strategy either in level change, speed change or heading change. After that, we 


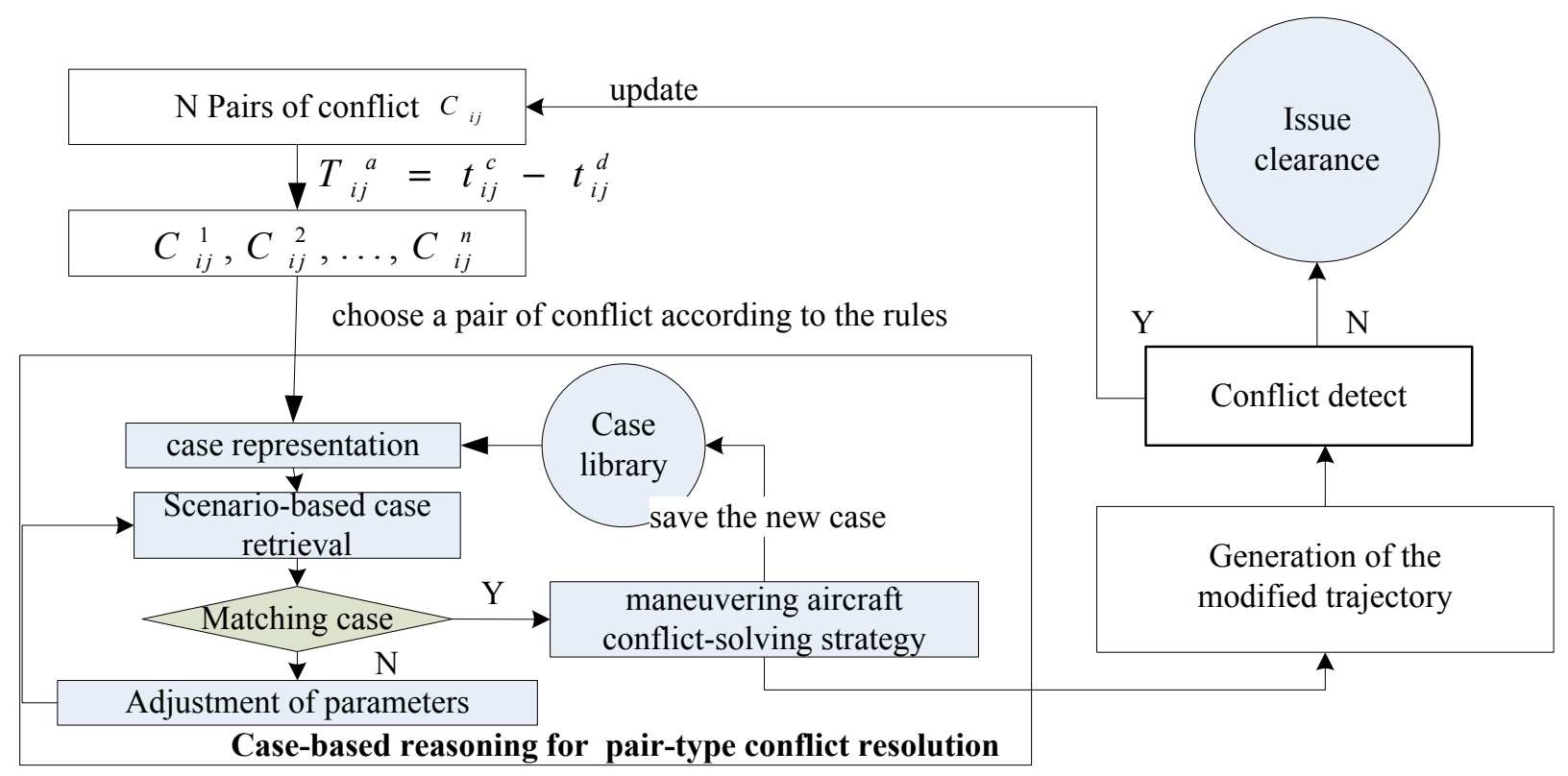

Fig. (3). Research approach of multi-aircraft conflicts resolution via Case-based Reasoning.

continue to detect other new conflicts with other aircrafts. The research approach is shown in Fig. (3).

\section{MODELLING OF EN-ROUTE FLIGHT CONFLICT RESOLUTION VIA CASE-BASED REASONING}

Unlike most problem solving methodologies in artificial intelligences (AI), CBR is a memory based and from cognitive science research on human memory, thus reflecting human use of remembered problems and solutions can be regarded as a starting point for new problem solving.

Solving a problem by CBR involves obtaining a problem description, measuring the similarity of the current problem to previous problems stored in a case base (or memory) with their known solutions, retrieving one or more similar cases, and attempting to reuse the solution of one of the retrieved cases, possibly after adapting it to account for differences in problem descriptions. The solution proposed by the system is then evaluated by being applied to the initial problem or assessed by a domain expert. Following revision of the proposed solution if required in light of its evaluation, the problem description and its solution can then be retained as a new case, and the system has learned to solve a new problem [17]. In [18] classic model of the problem solving cycle in CBR consists of four individual tasks separated as retrieve, reuse, revise and retain known as the 4 REs.

\subsection{Representation of Conflict Resolution Knowledge}

In this paper, representation of conflict resolution case is the key to realizing the conflict resolution via case-based reasoning. In order to describe a suitable conflict resolution case knowledge, the case features should be identified and the relations between these features should be established properly. Here, we adopt a framework-based method for representing the case knowledge.
The framework representation method was firstly proposed by American artificial intelligence scholar Minsky in 1975. It is a method for structured representation of knowledge developed on the basis of framework theory and is applicable to represent multiple types of knowledge. A basic view of framework theory is that the human brain has stored a large number of typical situations, and when confronted with a new situation, the brain will select from the memory a basic knowledge structure called framework, whose content varies with the new situation, forms an understanding of the new situation and stores it into the memory. The basic structure of a framework consists of a number of "slots", and each slot may, depending on the actual requirements, be divided into a number of "sides". The slot is used for description of features of the object while a side is used for description of one property of the features. The values in slot and a side are called slot value and side value respectively.

As the controllers normally handle a flight conflict based on the separation standard, it is adequate to proceed from separation minima and ATC procedures during the study of case framework. Therefore, we followed a long-term communication with experts from the area control center of Central and South China ATM Bureau (CATMB-CS), and firstly summed up experts' experiences based on the method of rules extraction. Besides, as conflict resolution is closely linked to the aircraft's operation tendency, a static analytical method based on rule-based reasoning is inadequate. Therefore, the intention of flight is incorporated into representation of case knowledge. Finally, the framework structure for case representation is shown in Table $\mathbf{1}$ by inference based on domain knowledge. The features and properties of an enroute conflict pair mainly are the relative track angle between two aircrafts named $a$ and $b$, relative distance, current flight state like level, speed, type of aircraft and track change intention. Besides, we take some environment like 
Table 1. Case description based on the frame structure.

\begin{tabular}{|c|c|c|}
\hline Slot & Side & Description \\
\hline \multirow{2}{*}{ Environment } & A1 & wind, thunderstorm etc. (1-yes, 0 -no) \\
\hline & $\mathrm{A} 2$ & temporary flight activities, like SAR etc. (1-yes, 0 -no) \\
\hline Relative Angle & A3 & Relative Angle between aircraft $a$ and $b\left({ }^{\circ}\right)$ \\
\hline \multirow{4}{*}{ Relative Distance } & A4 & horizontal distance from $a$ to first common point $p(\mathrm{~km})$ \\
\hline & A5 & horizontal distance from $b$ to first common point $p(\mathrm{~km})$ \\
\hline & A6 & difference between Sides A4 and A5(m) \\
\hline & A7 & difference of levels between $a$ and $b(\mathrm{~m})$ \\
\hline \multirow{6}{*}{ Flight State of Aircraft $a$} & A8 & current track angle of $a\left({ }^{\circ}\right)$ \\
\hline & A9 & track change intention of $a\left({ }^{\circ}\right)$ \\
\hline & A 10 & current level of $a(\mathrm{~m})$ \\
\hline & A11 & level change intention of $a(\mathrm{~m})$ \\
\hline & A12 & airspeed of $a$ (Knot) \\
\hline & A13 & type of aircraft $a(1-\mathrm{L}, 2-\mathrm{M}, 3-\mathrm{H}, 4-\mathrm{S})$ \\
\hline \multirow{6}{*}{ Flight State of Aircraft $b$} & A 14 & current track angle of $b\left({ }^{\circ}\right)$ \\
\hline & A15 & track change intention of $b\left({ }^{\circ}\right)$ \\
\hline & A16 & current level of $b(\mathrm{~m})$ \\
\hline & A17 & level change intention of $b(\mathrm{~m})$ \\
\hline & A18 & airspeed of $b$ (Knt) \\
\hline & A19 & type of aircraft $b$ (1-L, 2-M, 3-H, 4-S) \\
\hline \multirow{3}{*}{ Solution } & $\mathrm{D}$ & the aircraft that should firstly take action (1- $a, 2-b)$ \\
\hline & $a$ Action & maneuver intention taken by $a$, including (level, heading and $\mathrm{r} / \mathrm{c}$ or $\mathrm{r} / \mathrm{d}$ ) \\
\hline & $b$ Action & maneuver intention taken by $b$, including (level, heading and $\mathrm{r} / \mathrm{c}$ or $\mathrm{r} / \mathrm{d}$ ) \\
\hline
\end{tabular}

weather and temporary activities like SAR in consideration. We collected 33 stored cases in the library after careful preselection by experienced air traffic controllers with an experience of more than 8 years.

\subsection{Case Retrieval and Similarity Assessment}

Because of the central role of retrieval in the CBR cycle, a considerable amount of research has focused on retrieval and similarity assessment [17]. Case retrieval is the process of searching the previous cases in the case library that can be used to solve the target problem. A retrieval distance $R$ is defined to reflect the similarity between the input problem description and a stored problem description. Commonly used similarity-based retrieval approaches are Nearest neighbor algorithm, Inductive method and Template retrieval.
In this paper, a Nearest neighbor algorithm and global similarity measure based case retrieval approach is selected due to the small size of the case library available for flight conflict cases. We adopt hierarchical retrieval to increase retrieval speed. The source cases are filtered against constraint parameters before nearest neighbor algorithm is applied to calculate the degree of similarity between the target case and the source cases.

\section{(1) Case filtering}

For the purpose of reducing complexity and improving calculation efficiency, during the process of case retrieval we firstly look at $A 1, A 2$ and filter those previous cases which were influenced by specific environmental conditions like severe weather or temporary flight activities. 


\section{(2) Similarity measure}

Similarity measure between the target case and the previously stored cases in the library includes local similarity and global similarity measure. In our paper, each case represented a set of attribute-value features as $A 3, A 4, \ldots, A 19$, thus a local similarity measure is defined for each attribute of $A 3, A 4, \ldots, A 19$. While global similarity measure computed as a weighted average of the local similarities. The weights assigned to case attributes allow them to have varying degrees of importance and may be selected by a domain expert or user, or other methods. In this paper the weights are initially determined by the domain expert.

The detailed process of similarity assessment is described as follows.

Step 1. Calculate local similarity.

As the local attribute features $A 3, A 4, \ldots, A 19$ are classified by determinate features and indeterminate features, a pre-treatment is needed to make them in the same environment before calculating the global similarity.

With respect to an enumerated features such as $A 13$ and $A 19$, namely the type of aircraft, according to the level of wake turbulence such as Super Heavy, Heavy, Medium and Light. we give the degree of similarity by function as follows.

$\operatorname{sim}\left(L_{k}, X_{k}\right)= \begin{cases}1 & \text { if type of aircraft is same } \\ 0.5 & \text { if type of aircraft is near } \\ 0 & \text { others }\end{cases}$

Here, $X_{k}$ is the value of feature $k$ of the target case $X, L_{k}$ is the value of feature $k$ of the previous case $L$ in the stored case library, and $\operatorname{sim}\left(L_{k}, X_{k}\right)$ means the local similarity between $L_{k}$ and $X_{k}$.

With respect to numerical features, the following function is defined as follows.

$\operatorname{sim}\left(L_{k}, X_{k}\right)=1-\frac{\left|L_{k}-X_{k}\right|}{\max \left(L_{k}, X_{k}\right)}$

here, the $\max \left(L_{k}, X_{k}\right)$ is $X_{k}$ or $L_{k}$, whichever is greater.

Step 2. Calculate global similarity.

We use function (3) to calculate the global similarity.

$\operatorname{sim}(L, X)=\frac{\sum_{k=1}^{n}\left(w_{k} \times \max \left(L_{k}, X_{k}\right)\right)}{\sum_{k=1}^{n} w_{k}}$

in which $w_{k}$ is the weight of feature $k$, and $n$ is the number of features.
Calculation of weight $w_{k}$ is very important. In this paper, the initial weights of a Slot are determined by the domain expert through an order relation analysis method. Suppose there are a set of features $a_{1}, a_{2}, \ldots, a_{m}$, here $m$ is the number of Slot features. Firstly, the domain experts determine the most important feature marked as $a_{1}^{*}$, and then choose the second most important one marked as $a_{2}^{*}$. Follow this principle, after $n-1$ times of selection, finally it comes out an order of $a_{1}^{*}>a_{2}^{*}>a_{3}^{*}>\ldots>a_{m}^{*}$. Secondly, experts determine the relative importance between $a_{k-1}^{*}$ and $a_{k}^{*}$, and this can be labeled as $r_{k}$ with a relation defined by function (4), and the value of $r_{k}$ is referred to in Table 2.

Table (2). Reference value of $r_{k}$.

\begin{tabular}{|c|c|}
\hline$r_{k}$ & Meaning \\
\hline \hline 0 & $x_{k}$ is the same important with $x_{k-1}$ \\
\hline 2 & $x_{k}$ is slightly important with $x_{k-1}$ \\
\hline 4 & $x_{k}$ is more important with $x_{k-1}$ \\
\hline 6 & $x_{k}$ is largely important with $x_{k-1}$ \\
\hline 8 & $x_{k}$ is extremely important with $x_{k-1}$ \\
\hline
\end{tabular}

Table 3. Initial weights of Slot.

\begin{tabular}{|c|c|}
\hline Features & Weight \\
\hline \hline Relative Distance & 0.2756 \\
\hline Relative Angel & 0.3307 \\
\hline Flight State of Aircraft $a$ & 0.1969 \\
\hline Flight State of Aircraft $b$ & 0.1969 \\
\hline
\end{tabular}

$\frac{w_{k-1}}{w_{k}}=r_{k}$

here $k=m, m-1, m-2, \ldots, 3,2$.

The weight of $x_{m}^{*}$ is the less important one, it is determined by function (5).

$w_{m}=\left(1+\sum_{k=2}^{m} \prod_{i=k}^{m} r_{i}\right)^{-1}$ 
Table 4. Ten test cases of aircraft test scenario data.

\begin{tabular}{|c|c|c|c|c|c|c|c|c|c|c|c|c|c|c|c|c|c|}
\hline Case id & $\mathbf{A 3}$ & A4 & A5 & A6 & A7 & A8 & A9 & A10 & A11 & A12 & A13 & A14 & A15 & A16 & A17 & A18 & A19 \\
\hline $\mathrm{T} 1$ & 55 & 35 & 20 & 15 & 600 & 90 & 0 & 10100 & -600 & 840 & 2 & 130 & 0 & 9500 & 600 & 790 & 2 \\
\hline $\mathrm{T} 2$ & 50 & 35 & 20 & 15 & 0 & 90 & 0 & 10100 & 0 & 840 & 2 & 130 & 0 & 10100 & 0 & 790 & 2 \\
\hline $\mathrm{T} 3$ & 47 & 35 & 20 & 15 & 1200 & 90 & 0 & 10100 & -600 & 840 & 2 & 130 & 0 & 8900 & 1200 & 790 & 2 \\
\hline $\mathrm{T} 4$ & 46 & 35 & 35 & 0 & -450 & 90 & 0 & 9500 & 0 & 950 & 3 & 130 & 0 & 9950 & -1050 & 790 & 2 \\
\hline T5 & 45 & 35 & 35 & 0 & -1050 & 90 & 0 & 8900 & 0 & 950 & 3 & 130 & 0 & 9950 & -1050 & 790 & 2 \\
\hline T6 & 40 & 40 & 40 & 0 & 0 & 90 & 0 & 9500 & 0 & 950 & 3 & 130 & 0 & 9500 & 0 & 790 & 2 \\
\hline $\mathrm{T} 7$ & 30 & 42 & 24 & 18 & 600 & 180 & 0 & 5700 & 0 & 690 & 2 & 180 & 0 & 5100 & 0 & 650 & 2 \\
\hline $\mathrm{T} 8$ & 20 & 42 & 24 & 18 & 200 & 180 & 60 & 5100 & 0 & 650 & 2 & 180 & 0 & 4900 & 200 & 640 & 2 \\
\hline T9 & 10 & 25 & 7 & 18 & 1320 & 180 & 60 & 6300 & 0 & 650 & 2 & 180 & 0 & 4980 & 120 & 640 & 2 \\
\hline T10 & 0 & 42 & 24 & 18 & 720 & 180 & 0 & 5700 & 0 & 650 & 2 & 240 & -60 & 4980 & 120 & 640 & 2 \\
\hline
\end{tabular}

We know the $w_{m}$ and a set of $r_{k}$, then we know other $w_{k}$ by function (6)

$w_{k-1}=r_{k} \times w_{k}$

here, $k=m, m-1, \mathrm{~K}, 3,2$.

We distributed the questionnaires to ten experts, eight of which is valid. After calculation through the order relation analysis method it can be found that the suggested weight of each features tend to be stable after the number of expert is bigger than five, and finally the initial weights of Slot features are shown in Table $\mathbf{3}$.

\section{SIMULATION AND RESULT}

In order to test the performance of the proposed CBRbased conflict resolution model, to see whether it can produce the solution adapting to the reality, a specification of 10 synthetic traffic scenarios has been designed as Table $\mathbf{4}$, and 33 aircrafts scenarios were successfully executed by the controllers in the en-route control center of CATM-CS are stored in the case library. In this test, we suppose that the influence of environment is not considered and each Side feature has the same contribution to the upward Slot feature. The results of case retrieval are shown by Table 5 . The best matching case is marked by an underline. For T4 and T5, there are two best matching source cases; the reason is because we don't take the features A1 and A2 into consideration in this test. The result is well accepted by the air traffic controller.

To test the performance of the proposed CBR-based conflict resolution model in the presence of increment of the number of stored cases, we designed another trial. A new case is designed as a target case with a set of features

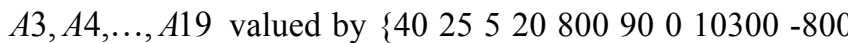

$84021300950007902\}$. In a target case, two aircrafts will converge at the same point, one's call-sign is RITA01 which is from flight level $10300 \mathrm{~m}$ descending to $9500 \mathrm{~m}$ with a heading of 090, the other's call-sign is RITA02 which is maintaining its flight level $9500 \mathrm{~m}$ with a heading of 130 . RITA01 is $5 \mathrm{~km}$ away from the crossing point and RITA02 is $25 \mathrm{~km}$ away from the crossing point, see Fig. (4), then, the 1 to 32 cases in the case library will be separately chosen as stored cases by 4 times in an increment of 8 cases. Matlab is used for programming and calculation. The results of the trial are shown in Table 6. We can see that along with the increase in the number of cases in the case library, the number of found cases increases, the value of maximum similarity $\operatorname{sim}$ also increases. For the target case, through reasoning by $\mathrm{CBR}$, it has the greatest similarity with the $11^{\text {th }}$ case situation in the case library, so the recommended strategy of conflict resolution for target case is: to instruct RITA01 to stop descent immediately and turn left 90 degrees and then instruct RITA02 to descend to $8900 \mathrm{~m}$. The resolution strategy is illustrated in Fig. (4).

\section{CONCLUSIONS}

On the basis of analyzing conflict resolution strategy operated by controllers from the cognitive perspective, this paper proposes a knowledge-driven approach for resolving en-route conflict in En-route STCA situation. By means of the rolling strategy, the problem of multiple conflicts among en-route aircraft under a complex environment is simplified into a set of sub-problem of pairwise conflicts. Instead of rule-based reasoning, the CBR technique from $\mathrm{AI}$ is applied, the study is not limited to getting descriptive success experience. Instead, it abstracts controllers' successful experiences, extracts rules and scenarios from the controllers' conflict 
Table 5. Results of global similarity assessment.

\begin{tabular}{|c|c|c|c|c|c|c|c|c|c|c|}
\hline Case id & T1 & $\mathbf{T} 2$ & T3 & T4 & T5 & T6 & $\mathbf{T 7}$ & T8 & T9 & T10 \\
\hline $\mathrm{S} 1$ & 0.50 & 0.53 & 0.51 & 0.46 & 0.45 & 0.50 & 0.85 & 0.89 & 0.81 & $\underline{0.95}$ \\
\hline $\mathrm{S} 2$ & 0.50 & 0.51 & 0.49 & 0.42 & 0.41 & 0.45 & 0.79 & 0.86 & $\underline{0.90}$ & 0.85 \\
\hline $\mathrm{S} 3$ & 0.52 & 0.53 & 0.52 & 0.46 & 0.45 & 0.50 & 0.85 & $\underline{0.93}$ & 0.84 & 0.91 \\
\hline S4 & 0.54 & 0.56 & 0.53 & 0.49 & 0.48 & 0.53 & $\underline{0.87}$ & 0.90 & 0.82 & 0.92 \\
\hline S5 & 0.78 & 0.80 & 0.77 & 0.91 & 0.89 & $\underline{0.97}$ & 0.58 & 0.57 & 0.49 & 0.51 \\
\hline S6 & 0.77 & 0.79 & 0.77 & $\underline{0.96}$ & 0.93 & 0.92 & 0.57 & 0.55 & 0.50 & 0.50 \\
\hline S7 & 0.77 & 0.79 & 0.77 & 0.96 & 0.93 & 0.92 & 0.57 & 0.55 & 0.50 & 0.50 \\
\hline S8 & 0.92 & 0.87 & $\underline{0.95}$ & 0.77 & 0.76 & 0.78 & 0.68 & 0.62 & 0.65 & 0.62 \\
\hline S9 & 0.94 & $\underline{0.97}$ & 0.91 & 0.83 & 0.80 & 0.82 & 0.65 & 0.64 & 0.58 & 0.58 \\
\hline $\mathrm{S} 10$ & $\underline{0.97}$ & 0.93 & 0.94 & 0.81 & 0.79 & 0.80 & 0.68 & 0.64 & 0.61 & 0.61 \\
\hline S11 & 0.90 & 0.85 & 0.88 & 0.75 & 0.73 & 0.74 & 0.63 & 0.58 & 0.65 & 0.57 \\
\hline$\ldots$ & $\ldots$ & $\ldots$ & $\ldots$ & $\ldots$ & $\ldots$ & $\ldots$ & $\ldots$ & $\ldots$ & $\ldots$ & $\ldots$ \\
\hline S33 & 0.36 & 0.40 & 0.34 & 0.35 & 0.33 & 0.35 & 0.18 & 0.19 & 0.17 & 0.18 \\
\hline
\end{tabular}

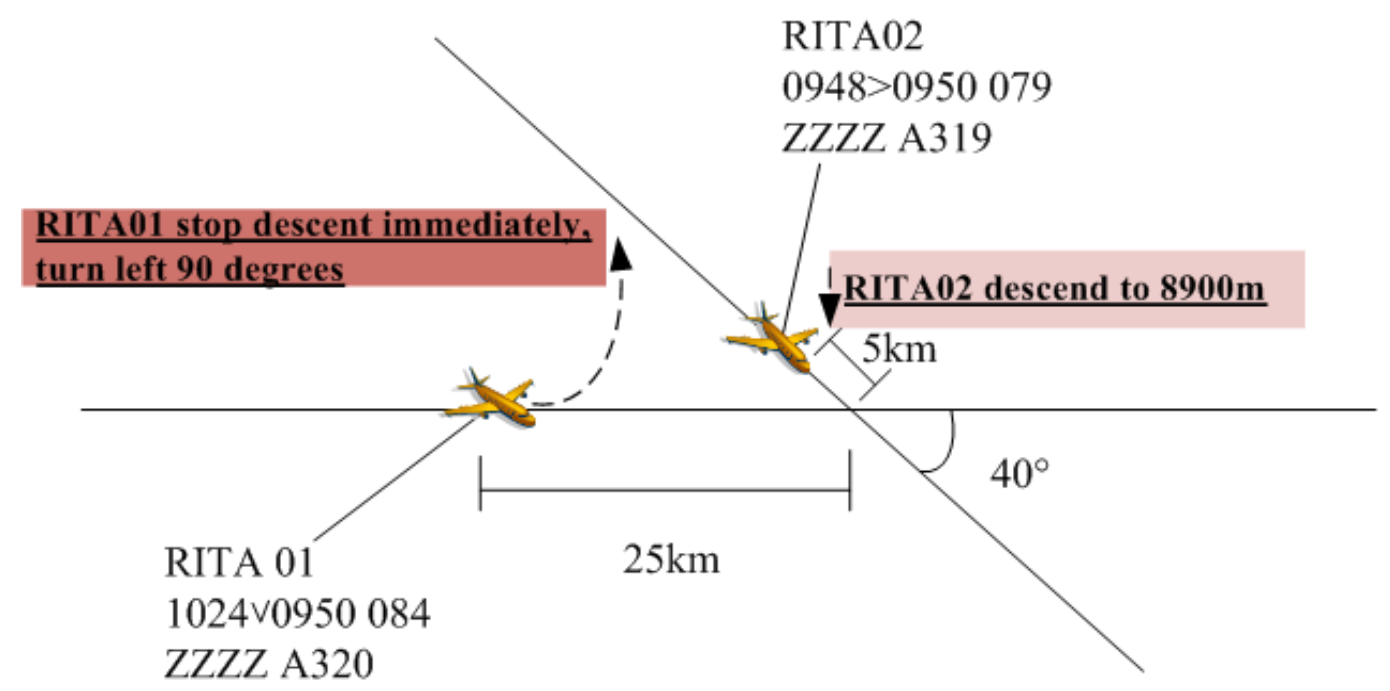

\section{Label illustratation}

Callsign

Present FL| tendency target| FL Ground speed (*10)

Destination code| type of aircraft

Fig. (4). Automated conflict resolution scenario based on CBR. 
Table 6. Results of the test under different number of stored cases.

\begin{tabular}{|c|c|c|c|c|}
\hline Test id & No. of Stored Cases & No. of Found Cases & Max. of sim & Elapsed Time $(s)$ \\
\hline \hline 1 & 8 & 1 & 0.8464 & 0.005912 \\
\hline 2 & 16 & 7 & 0.9366 & 0.007909 \\
\hline 3 & 24 & 7 & 0.9679 & 0.008880 \\
\hline 4 & 34 & 13 & 0.9791 & 0.010006 \\
\hline
\end{tabular}

resolution strategies, and applies them to scientific reasoning.

In the present of STCA, controllers are easily losing situation awareness, especially those who never had such emergency experiences before. An automated conflict resolution can help them to handle the strict emergency in a short range time, which can greatly improve the safety level at the front line. Further research should concentrate on reducing the unnecessary features in the Side level by using a Rough-set theory, so as to reduce the case search volume and increase the searching speed.

\section{CONFLICT OF INTEREST}

The author confirms that this article content has no conflict of interest.

\section{ACKNOWLEDGEMENTS}

Gratitude should go to controllers of Central and South China ATM Bureau, who helped collect cases, as well as anonymous reviewers for their constructive revision suggestions. This work was supported by National Natural Science Foundation (No.U1333116, No.61039001), Fundamental research funds for the Central Universities Civil Aviation University of China special Grant 3122013D013.

\section{REFERENCES}

[1] J. Krozel, T. Mueller and G. Hunter, "Free flight conflict detection and resolution analysis," In: AIAA conference and exhibit in guidance, navigation, and control, 1996.

[2] J. Krozel and M. Peters, "Strategic conflict detection and resolution for free flight," In: IEEE Proc. 36th IEEE Conf. Decision Control 2, pp. 1822-1828,1997.

[3] N. Durand, J. Alliot, "Optimal resolution of en route conflicts", In: Proc. 1st USA/Europe Seminar, 1997.

[4] G. Granger, N. Durand, J. Alliot, "Optimal resolution of en route conflicts", In: 4th ATM R and D Seminar, 2001.
[5] K. D. Bilimoria, "A geometric optimization approach to aircraft conflict resolution", In: AIAA conference and exhibit in guidance, navigation, and control", Reston: VA: USA, pp. 14-17, 2000.

[6] R. A. Paielli, " Modeling maneuver dynamics in air traffic conflict resolution," J. Guid. Control Dyna., vol. 26, no. 3, pp.407-415, 2003

[7] R. A. Paielli, "Tactical conflict resolution using vertical maneuvers in en route airspace", J. Aircraft, vol. 45, no. 6, pp. 2111-2119, 2008

[8] H. Erzberger, "Automated conflict resolution for air traffic control National Aeronautics and Space Administration," Ames Res. Center, 2005

[9] H. Erzberger, "The automated airspace concept," In: 4th USA/Europe Air Traffic Management R\&D Seminar.

[10] H. Erzberger, T. Lauderdale and Y. Chu, "Automated conflict resolution, arrival management and weather avoidance for ATM," In: 27th Int. Cong. Aeronaut. Sci., Nice: France, 2010.

[11] H. Erzberger and K. Heere, "Algorithm and operational concept for resolving short-range conflicts," In: Proc. Instit. Mech. Eng., Part G: J. Aerospace Eng., vol. 224, no. 2 pp. 225-243, 2010

[12] D. R. Isaacson and J. E. Robinson, "A knowledge-based conflict resolution algorithm for terminal area air traffic control advisory generation," In: AIAA Guidance, Navigation, and Control Conference, Montreal: Canada, 2001

[13] B. S. Yang, T. Han, and Y. S. Kim, "Integration of art-kohonen neural network and case-based reasoning for intelligent fault diagnosis," Expert Syst. Appl., vol. 26, no. 3, pp. 387-395, 2004.

[14] M. J. Huang, M. Y. Chen and S. C. Lee, "Integrating data mining with case-based reasoning for chronic diseases prognosis and diagnosis," Expert Syst. Appl., vol. 32, no. 3, pp. 856-867, 2007

[15] S. Begum, M. Ahmed, P. Funk, N. Xiong, and M. Folke, "Casebased reasoning systems in the health sciences: a survey of recent trends and developments," In: IEEE Transact. Sys. Man, Cybern., Part C: Appl. Rev., vol. 41, no. 4, pp. 421-434,2011.

[16] A. Majumdar and J. Polak, "Estimating capacity of Europe's airspace using a simulation model of air traffic controller workload", J. Transport. Res. Board, vol. 1744, no. 1, pp. 30-43,2001

[17] R. Lopez, D. Mantaras, D. McSherry, D. Bridge, D. Leake, B. Smyth, S. Craw, B. Faltings, M. L. Maher, M. T. Cox, K. Forbus, M. keane, A. Aamodt and I. Watson, "Retrieval, reuse, revision and retention in case-based reasoning," The Knowledge Eng. Rev., vol. 20, no. 3, pp. 215-240, 2005.

[18] A. Aamodt and E. Plaza, "Case-based reasoning: Foundational issues, methodological variations, and system approaches", AI Commun., vol. 7, no. 1, pp. 39-59, 1994.

(C) Man Liang; Licensee Bentham Open.

This is an open access article licensed under the terms of the Creative Commons Attribution Non-Commercial License (http://creativecommons.org/licenses/ by-nc/4.0/) which permits unrestricted, non-commercial use, distribution and reproduction in any medium, provided the work is properly cited. 